\title{
Smooth Cores of Lipschitz Flows
}

\author{
By
}

\author{
Derek W. ROBINSON*
}

\begin{abstract}
We prove that the smooth elements of an abelian $C^{*}$-dynamical system constitute a core of the associated Lipschitz flows.
\end{abstract}

\section{§1. Introduction}

The purpose of this note is to continue the analysis of Lipschitz flows developed in [2]. The main object of investigation in [2] was a ${ }^{*}$-derivation $\delta$ associated with an abelian $C^{*}$-dynamical system and determined by a function $\lambda$. Two principal results were obtained. First it was shown that if $\lambda$ satisfies a Lipschitz condition then $\delta$ has a unique generator extension. Second if $\lambda$ is differentiable then the generator extension is the closure of $\delta$ restricted to a class of smooth elements of the system. In this note we prove that this second conclusion in fact follows from the Lipschitz condition. The proof of this observation relies heavily on the techniques developed in [2] combined with a semigroup convergence argument. As a preliminary we recall some definitions and notation.

Let $\mathscr{A}$ denote an abelian $C^{*}$-algebra and $t \in \mathbb{R} \rightarrow \sigma_{t}$ a $C_{0}$-group of *-automorphisms of $\mathscr{A}$ with generator $\delta_{0}$. Define $\mathscr{A}_{n}=D\left(\delta_{0}^{n}\right)$ and $\mathscr{A}_{\infty}=\cap_{n \geq 1} \mathscr{A}_{n}$. Now $\mathscr{A}$ can be identified with $C_{0}(X)$, the continuous functions over the spectrum $X$ of $\mathscr{A}$ which vanish at infinity, and one can associate with $\sigma$ a continuous one-parameter group $S$ of homeomorphisms of $X$ such that

$$
\left(\sigma_{t} f\right)(\omega)=f\left(S_{t} \omega\right)
$$

Communicated by H. Araki October 18, 1985. Revised December 17, 1985.

*Department of Mathematics, Institute of Advanced Studies, Australian National University, Canberra 
for all $t \in R, \omega \in X$, and $f \in C_{0}(X)$. Next define the period $p(\omega)$ of a point $\omega \in X$ with respect to the group $S$ by

$$
p(\omega)=\inf \left\{t>0 ; S_{t} \omega=\omega\right\}
$$

and the frequency $\nu(\omega)$ of $\omega$ by $\nu(\omega)=1 / p(\omega)$. Thus $\omega$ is in the fixed point set

$$
X_{0}=\left\{\omega ; S_{t} \omega=\omega \text { for all } t \in R\right\}
$$

if, and only if, $p(\omega)=0$ or $\nu(\omega)=\infty$. Finally if $\lambda \in C\left(X \backslash X_{0}\right)$ is real define a ${ }^{*}$-derivation $\delta=\lambda \delta_{0}$ on $\mathscr{A}$ by

$$
(\delta f)(\omega)= \begin{cases}\lambda(\omega)\left(\delta_{0} f\right)(\omega) & \text { if } \omega \in X \backslash X_{0} \\ 0 & \text { if } \omega \in X_{0}\end{cases}
$$

with the domain $D(\delta)$ of $\delta$ consisting of those $f \in D\left(\delta_{0}\right)$ for which the right hand side is in $C_{0}(X)$. Now we can state our principal result.

Theorem 1.1. Let $\lambda \in C\left(X \backslash X_{0}\right)$ be a real function satisfying the following two conditions.

1. Low frequency boundedness-For any compact subset $C \subseteq X$ there exists an $\varepsilon>0$ such that $\lambda$ is uniformly bounded on

$$
C \cap\{\omega \in X ; \nu(\omega)<\varepsilon\}
$$

2. Lipschitz property - For each $\omega \in X \backslash X_{0}$ and all $t \in \boldsymbol{R}$

$$
\left|\lambda\left(S_{t} \omega\right)-\lambda(\omega)\right| \leq K(\nu(\omega))|t|
$$

where $K: \boldsymbol{R}_{+} \rightarrow \boldsymbol{R}_{+}$is bounded on bounded intervals.

It follows that the closure of $\delta=\lambda \delta_{0}$ restricted to $\mathscr{A}_{\infty} \cap D(\delta)$ generates a

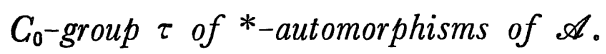

Theorem 2.6 of [2] established that under the hypotheses of the theorem $\delta$ has a unique generator extension $H$ and the new element is that $\mathscr{A}_{\infty} \cap D(\delta)$ is a core of $H$. This will be proved in the next section.

\section{§2. Generator Cores}

The proof of the core property in Theorem 1.1 is a combination of the results of [2], a regularization technique, and a semigroup convergence argument. 
We begin by introducing regularizations $\lambda_{\alpha}, \alpha>0$, of $\lambda$ as follows. Let $g$ be a positive differentiable function with compact support in $[-1,1]$, and with total integral one. Then define

$$
\lambda_{\alpha}(\omega)=\int d t g(t) \lambda\left(S_{\alpha t} \omega\right) .
$$

It follows immediately that $\lambda_{\alpha}$ satisfies both conditions of the theorem and hence each of the associated derivations $\delta_{\alpha}=\lambda_{\alpha} \delta_{0}$ has a unique generator extension $H_{\alpha}$. Now if $\tau^{\alpha}$, and $\tau$, denote the groups generated by $H_{\alpha}$, and $H$, respectively, then we prove that $\tau^{\alpha}$ converges strongly to $\tau$ as $\alpha \rightarrow 0$. The proof is based on the following general criterion.

Proposition 2.1. Let $\tau^{\alpha}$, $\tau$ be $C_{0}$-groups of $*_{\text {-automorphisms of the }}$ $C^{*}$-algebra $C_{0}(X)$ and $T^{\alpha}, T$ the associated groups of homeomorphisms of $X$.

The following conditions are equivalent;

1. $\lim _{\alpha}\left|\tau_{t}^{\alpha} f-\tau_{t} f\right| \mid=0, \quad t \in \mathbb{R}, f \in C_{0}(X)$,

2. If $\omega_{\alpha} \rightarrow \omega$ then

$$
\lim _{\alpha} T_{t}^{\alpha} \omega_{\alpha}=T_{t} \omega, \quad t \in \mathbb{R} .
$$

3. If $\omega_{\alpha} \rightarrow \omega$ and $\left\{T_{t}^{\alpha} \omega_{\alpha}\right\}$ has a convergent subnet $\left\{T_{t}^{\alpha^{\prime}} \omega_{\alpha^{\prime}}\right\}$ then

$$
\lim _{\alpha^{\prime}} T_{t}^{\alpha^{\prime}} \omega_{\alpha^{\prime}}=T_{t} \omega, \quad t \in \mathbb{R} 。
$$

Proof. $1 \Rightarrow 2$. Let $f \in C_{0}(X)$ then

$$
\left|f\left(T_{t}^{\alpha} \omega_{\alpha}\right)-f\left(T_{t} \omega\right)\right| \leq\left\|\tau_{t}^{\alpha} f-\tau_{t} f\right\|+\left|\left(\tau_{t} f\right)\left(\omega_{\alpha}\right)-\left(\tau_{t} f\right)(\omega)\right| .
$$

Therefore

$$
\lim _{\alpha}\left|f\left(T_{t}^{\alpha} \omega_{\alpha}\right)-f\left(T_{t} \omega\right)\right|=0
$$

and since $X$ is locally compact

$$
\lim _{\alpha} T_{t}^{\alpha} \omega_{\alpha}=T_{t} \omega .
$$

$2 \Rightarrow 1$. Assume Condition 1 is false, i.e. there exists an $f \in C_{0}(X)$ and $t \in \boldsymbol{R}$ such that

$$
\limsup _{\alpha}\left\|\tau_{t}^{\alpha} f-\tau_{t} f\right\|=2 \varepsilon>0 .
$$

Hence there must exist a subnet, also denoted by $\tau_{t}^{\alpha} f$, and corresponding $\omega_{\alpha}$ such that

$$
\left|\left(\tau_{t}^{\alpha} f\right)\left(\omega_{\alpha}\right)-\left(\tau_{t} f\right)\left(\omega_{\alpha}\right)\right|>\varepsilon,
$$


or, equivalently,

$$
\left|f\left(T_{t}^{\alpha} \omega_{\alpha}\right)-f\left(T_{t} \omega_{\alpha}\right)\right|>\varepsilon_{0}
$$

But there also exists a compact set $K \subseteq X$ such that $|f(\omega)|<\varepsilon / 2$ if $\omega \notin K$. Therefore for each $\alpha$ one has either $T_{t} \omega_{\alpha} \in K$ or $T_{t}^{\alpha} \omega_{\alpha} \in K$ and since $K$ is compact there is a subnet such that either $T_{t} \omega_{\alpha^{\prime}} \rightarrow T_{t} \omega \in K$ or $\rho_{\alpha^{\prime}}=T_{t}^{\alpha^{\prime}} \omega_{\alpha^{\prime}} \rightarrow \rho \in K$.

Consider the first possibility. Then $\omega_{\alpha^{\prime}} \rightarrow \omega$ and $T_{t}^{\alpha^{\prime}} \omega_{\alpha^{\prime}} \rightarrow T_{t} \omega$ by Condition 2. But this gives

$$
\varepsilon \leq \lim _{\alpha^{\prime}}\left|f\left(T_{t}^{\alpha^{\prime}} \omega_{\alpha^{\prime}}\right)-f\left(T_{t} \omega_{\alpha^{\prime}}\right)\right|=0
$$

which is an inconsistency.

Now consider the second possibility. Then $T_{-t}^{\alpha^{\prime} \rho_{\alpha^{\prime}} \rightarrow T_{-t} \rho}$ by Condition 2 or, alternatively stated, $\omega_{\alpha^{\prime}} \rightarrow T_{-t} \rho$. Then one has

$$
\varepsilon \leq \lim _{\alpha^{\prime}}\left|f\left(\rho_{\alpha^{\prime}}\right)-f\left(T_{t} T_{-t}^{\alpha_{t}^{\prime}} \rho_{\alpha^{\prime}}\right)\right|=0
$$

which is again inconsistent.

Therefore Condition 1 must be valid and $2 \Rightarrow 1$. But this argument also establishes $1 \Rightarrow 3$ so the proof is complete.

Now we apply this convergence criterion to the groups $\tau$, and $\tau^{\alpha}$, generated by the extensions $H$, and $H_{\alpha}$, of $\delta=\lambda \delta_{0}$, and $\delta_{\alpha}=\lambda_{\alpha} \delta_{0}$.

First consider the group $T^{\alpha}$ of homeomorphisms of $X$ associated with $\tau^{\alpha}$. It follows from the construction in [2] that

$$
T_{t}^{\alpha} \omega=S_{x_{\omega}^{\alpha}(t)} \omega
$$

where $x_{\omega}^{\alpha}$ is the unique solution of the initial-value problem

$$
\begin{aligned}
& \dot{x}_{\omega}^{\alpha}(t)=\lambda_{\alpha, \omega}\left(x_{\omega}^{\alpha}(t)\right), \\
& x_{\omega}^{\alpha}(0)=0,
\end{aligned}
$$

and

$$
\lambda_{\alpha, \omega}(t)=\lambda_{\alpha}\left(S_{t} \omega\right)
$$

Now suppose $\omega_{\alpha} \rightarrow \omega$ and $\nu(\omega)<\infty$. Since $\nu$ is upper-semicontinuous, by Lemma 2.3 of [2], one has lim sup $\nu\left(\omega_{\alpha}\right) \leq \nu(\omega)$ and hence one can assume that $\nu\left(\omega_{\alpha}\right) \leq 1+\nu(\omega)$. Next since $\lambda\left(\omega_{\alpha}\right) \rightarrow \lambda(\omega)$ one can also assume $\left|\lambda\left(\omega_{\alpha}\right)\right| \leq 1+|\lambda(\omega)|$. Hence setting $\lambda_{\omega}(t)=\lambda\left(S_{t} \omega\right)$ one has

$$
\begin{aligned}
\left|\lambda_{\alpha, \omega_{\alpha}}(t)-\lambda_{\alpha, \omega_{\alpha}}(0)\right| & \leq \int d s g(s)\left|\lambda\left(S_{\alpha s+t} \omega_{\alpha}\right)-\lambda\left(S_{\alpha s} \omega_{\alpha}\right)\right| \\
& \leq|t| \int d s g(s) K\left(\nu\left(\omega_{\alpha}\right)\right)
\end{aligned}
$$


and

$$
\begin{aligned}
\left|\lambda_{\alpha, \omega_{\alpha}}(0)-\lambda_{\omega_{\alpha}}(0)\right| & \leq \int d s g(s)\left|\lambda\left(S_{\alpha s} \omega_{\alpha}\right)-\lambda\left(\omega_{\alpha}\right)\right| \\
& \leq \alpha \int d s|s| g(s) K\left(\nu\left(\omega_{\alpha}\right)\right) .
\end{aligned}
$$

Consequently there is a $K_{\omega}>0$ such that

$$
\left|\lambda_{\alpha, \omega_{\alpha}}(t)\right| \leq K_{\omega}(1+|t|)
$$

for $0<\alpha<1$ and all $t \in \mathbb{R}$. Similarly

$$
\left|\lambda_{\alpha, \omega}(t)\right| \leq K_{\omega}(1+|t|) \text {. }
$$

Now fix $t_{0}>0$, introduce

$$
M=\left(e^{K_{\omega} t_{0}}-1\right) / t_{0}
$$

and define

$$
M_{\alpha}=\sup \left\{\left|\lambda_{\alpha, \omega_{\alpha}}(t)-\lambda_{\alpha, \omega}(t)\right| ;|t| \leq M t_{0}\right\} 。
$$

It then follows by the proof of Observation 1 in Section 2 of [2] that

$$
\left|x_{\omega_{\alpha}}^{\alpha}(t)-x_{\omega}^{\alpha}(t)\right| \leq M_{\alpha}\left(e^{M|t|}-1\right) / M
$$

for all $|t| \leq t_{0}$. But

$$
\begin{aligned}
&\left|\lambda_{\alpha, \omega_{\alpha}}(t)-\lambda_{\alpha, \omega}(t)\right| \leq \int d s g(s)\left|\lambda\left(S_{\alpha s+t} \omega_{\alpha}\right)-\lambda\left(S_{\alpha s+t} \omega\right)\right| \\
& \underset{\alpha \rightarrow 0}{\longrightarrow} 0
\end{aligned}
$$

for each $t \in\left[-M t_{0}, M t_{0}\right]$ and since $\lambda_{\alpha, \omega_{\alpha}}$ and $\lambda_{\alpha, \omega}$ are both uniformly Lipschitz it follows that the convergence is uniform for $t$ in the interval $\left[-M t_{0}, M t_{0}\right]$. Therefore $M_{\alpha} \rightarrow 0$ as $\alpha \rightarrow 0$ and consequently

$$
\lim _{\alpha}\left|x_{\omega_{\alpha}}^{\alpha}(t)-x_{\omega}^{\alpha}(t)\right|=0 \text {. }
$$

Next remark that it follows from the differential equation for $x_{\omega}^{\alpha}$ and the similar equation

$$
\dot{x}_{\omega}(t)=\lambda_{\omega}\left(x_{\omega}(t)\right)
$$

for $x_{\omega}$ that

$$
\begin{aligned}
\left|x_{\omega}^{\alpha}(t)-x_{\omega}(t)\right| & \leq \int_{0}^{|t|} d r \int d s g(s)\left|\lambda_{\omega}\left(x_{\omega}^{\alpha}(r)+\alpha s\right)-\lambda_{\omega}\left(x_{\omega}(r)\right)\right| \\
& \leq K(\nu(\omega))\left\{\alpha|t| \int d s g(s)|s|+\int_{0}^{|t|} d r\left|x_{\omega}^{\alpha}(r)-x_{\omega}(r)\right|\right\} .
\end{aligned}
$$


Hence by iteration of this integral inequality

$$
\left|x_{\omega}^{\alpha}(t)-x_{\omega}(t)\right| \leq \alpha|t| \int d s g(s)|s| K(\nu(\omega)) \exp \{|t| K(\nu(\omega))\}
$$

and

$$
\lim _{\alpha \rightarrow 0}\left|x_{\omega}^{\alpha}(t)-x_{\omega}(t)\right|=0 .
$$

Combining these estimates one concludes that

$$
\begin{aligned}
\lim _{\alpha \rightarrow 0}\left|x_{\omega_{\alpha}}^{\alpha}(t)-x_{\omega}(t)\right| & \leq \lim _{\alpha \rightarrow 0}\left\{\left|x_{\omega_{\alpha}}^{\alpha}(t)-x_{\omega}^{\alpha}(t)\right|+\left|x_{\omega}^{\alpha}(t)-x_{\omega}(t)\right|\right\} \\
& =0 .
\end{aligned}
$$

It now follows by joint continuity of the $\operatorname{map}(t, \omega) \rightarrow S_{t} \omega$ that

$$
\begin{aligned}
\lim _{\alpha \rightarrow 0} T_{t}^{\alpha} \omega_{\alpha} & =\lim _{\alpha \rightarrow 0} S_{x_{\omega_{\alpha}}^{\alpha}(t)} \omega_{\alpha} \\
& =S_{x_{\omega^{(}}(t)} \omega=T_{t} \omega .
\end{aligned}
$$

This verifies Condition 2, or 3, of Proposition 2 for the case $\nu(\omega)<+\infty$.

Next suppose $\nu(\omega)=+\infty$ or, equivalently, $\omega \in X_{0}$ and also suppose $T_{t}^{\alpha} \omega_{\alpha}$, or a subnet, is convergent. Then either $\nu\left(\omega_{\alpha}\right) \rightarrow 0$ or $\nu\left(\omega_{\alpha^{\prime}}\right)$ is bounded away from zero for all $\alpha^{\prime}$ in some subnet. But if $\nu\left(\omega_{\alpha}\right) \rightarrow 0$ it follows from the low frequency boundedness condition that $\lambda\left(S_{t} \omega_{\alpha}\right)$ is bounded uniformly in $\alpha$ and $t$ for $0 \leq t \leq 1$. Therefore $\lambda_{\alpha}\left(\omega_{\alpha}\right)$ is bounded uniformly in $\alpha$ for $0<\alpha \leq 1$. Then using the Lipschitz condition one deduces as above that one has a bound

$$
\left|x_{\omega_{\alpha^{\prime}}}^{\alpha^{\prime}}(t)\right| \leq K
$$

for all small $t$ and all $\alpha^{\prime}$ in a subnet $\alpha^{\prime} \rightarrow 0$. Hence

$$
T_{t}^{\alpha^{\prime}} \omega_{\alpha^{\prime}}=S_{x_{\omega_{\alpha^{\prime}}{ }^{\prime}(t)}} \omega_{\alpha^{\prime}} \subseteq S_{[-K, K]} \omega_{\alpha^{\prime}}
$$

for small $t$. But since $\omega \in X_{0}$ and $(t, \omega) \rightarrow S_{t} \omega$ is jointly continuous it follows that

$$
\lim _{\alpha^{\prime}} T_{t}^{\alpha^{\prime}} \omega_{\alpha^{\prime}}=\omega \text {. }
$$

Finally if $\nu\left(\omega_{\alpha^{\prime}}\right)$ is bounded away from zero for some subnet, i.e. if $\nu\left(\omega_{\alpha^{\prime}}\right)>\varepsilon$ for all $\alpha^{\prime}$ then

$$
T_{t}^{\alpha^{\prime}} \omega_{\alpha^{\prime}} \subseteq S_{R} \omega_{\alpha^{\prime}}=S_{[0,1 / \varepsilon]} \omega_{\alpha^{\prime}}
$$

and hence $T_{t}^{\alpha^{\prime}} \omega_{\alpha^{\prime}} \rightarrow \omega$. But this implies $T_{t}^{\alpha} \omega_{\alpha} \rightarrow \omega$. Thus Condition 3 of Proposition 1 is valid under all circumstances. 
This completes the proof that $\tau_{t}^{\alpha}$ converges strongly to $\tau_{t}$ for each $t \in \mathbb{R}$. But then it is a standard consequence of the Trotter-Kato theorem on semigroup convergences that $\tau_{t}^{\alpha}$ converges to $\tau_{t}$ uniformly for $t$ in bounded intervals. In particular if $h \in C_{0}(\mathbb{R})$ has compact support and

$$
\tau_{h}^{\alpha}=\int d t h(t) \tau_{t}^{\alpha}
$$

then $\tau_{h}^{\alpha}$ converges strongly to $\tau_{h}$,

Next we need information on the domains of the derivations $\delta$ and $\delta_{\alpha}$. First following [2] we introduce the sets $\mathscr{D}_{n}, n=1,2, \ldots, \infty$, consisting of those $f \in \mathscr{A}_{n}$ with compact support for which $t \rightarrow f\left(S_{t} \omega\right)$ is constant if $\nu(\omega)$ is sufficiently large. It then follows from Lemma 2.7 of [2] that $\mathscr{D}_{\infty} \cap D(\delta)$ and $\mathscr{D}_{\infty} \cap D\left(\delta_{\alpha}\right)$ are both dense in $\mathscr{A}$. But in fact these sets are equal.

Lemma 2.2. For each $\alpha>0$ and $n=1,2, \ldots, \infty$, one has

$$
\mathscr{D}_{n} \cap D(\delta)=\mathscr{D}_{n} \cap D\left(\delta_{\alpha}\right)
$$

Proof. If $f \in \mathscr{D}_{n} \cap D(\delta)$ then

(*) $\quad \lambda_{\alpha}(\omega)\left(\delta_{0} f\right)(\omega)=\lambda(\omega)\left(\delta_{0} f\right)(\omega)+\int d s g(s)\left(\lambda\left(S_{\alpha s} \omega\right)-\lambda(\omega)\right)\left(\delta_{0} f\right)(\omega)$

for $\omega \in X \backslash X_{0}$. But since $f \in \mathscr{D}(\delta)$ the first term on the right hand side extends to an element of $C_{0}(X)$ vanishing on $X_{0}$. Now consider the second term. This is clearly a continuous function on $X \backslash X_{0}$ but it also is bounded by

$$
\alpha \int d s g(s)|s| K(\nu(\omega))\left|\left(\delta_{0} f\right)(\omega)\right|
$$

and this bound vanishes at infinity on $X \backslash X_{0}$. This follows because $f \in \mathscr{D}_{n}$ implies $\left(\delta_{0} f\right)(\omega)=0$ for $\nu(\omega)$ larger than some finite value $m$, $K$ is bounded on $[0, m]$, and $\delta_{0} f$ vanishes at infinity on $X \backslash X_{0}$. Now this establishes that the second term on the right hand side of $(*)$ extends to an element of $C_{0}(X)$ vanishing on $X_{0}$, which implies $f \in D\left(\delta_{\alpha}\right)$. Hence $\mathscr{D}_{n} \cap D(\delta) \subseteq \mathscr{D}_{n} \cap D\left(\delta_{\alpha}\right)$. But an almost identical argument gives the converse inclusion and consequently $\mathscr{D}_{n} \cap D(\delta)=$ $\mathscr{D}_{n} \cap D\left(\delta_{\alpha}\right)$. 
The next result is a direct consequence of Lemma 2.2 and Lemma 2.11 of [2] but at this point it is crucial to observe that $\lambda_{\alpha}$ is differentiable in the sense that the limit

$$
\begin{aligned}
\left(\delta_{0} \lambda_{\alpha}\right)(\omega) & =\lim _{t \rightarrow 0}\left(\lambda_{\alpha}\left(S_{t} \omega\right)-\lambda_{\alpha}(\omega)\right) / t \\
& =-\alpha^{-1} \int d s g^{\prime}(s) \lambda\left(S_{\alpha s} \omega\right)
\end{aligned}
$$

exists pointwise and is a continuous function of $\omega \in X \backslash X_{0}$.

Lemma 2.3. If $f \in \mathscr{D}_{\infty} \cap D(\delta)$ then $\tau_{t}^{\alpha} f \in \mathscr{D}_{1} \cap D(\delta)$, for all $t \in \boldsymbol{R}$ and $\alpha>0$. Moreover $\left(\delta_{0} \tau_{t}^{\alpha} f\right)(\omega)=0$ for $\omega \in X_{0}$ and

$$
\left(\delta_{0} \tau_{t}^{\alpha} f\right)(\omega)=\exp \left\{\int_{0}^{t} d s\left(\tau_{s}^{\alpha} \delta_{0} \lambda_{\alpha}\right)(\omega)\right\}\left(\tau_{t}^{\alpha} \delta_{0} f\right)(\omega)
$$

for $\omega \in X \backslash X_{0}$. Finally if $h \in C_{0}^{\infty}(\mathbb{R})$ has compact support then $\tau_{h}^{\alpha} f \in \mathscr{D}_{1} \cap$ $D(\delta)$.

Proof. Since $\lambda_{\alpha}$ is differentiable

$$
\tau_{t}^{\alpha}\left(\mathscr{D}_{\infty} \cap D\left(\delta_{\alpha}\right)\right) \subseteq \mathscr{D}_{1} \cap D\left(\delta_{\alpha}\right)
$$

by Lemma 2.11 (c) of [2]. But $\mathscr{D}_{n} \cap D\left(\delta_{\alpha}\right)=\mathscr{D}_{n} \cap D(\delta)$ by Lemma 2.2 and this establishes the first statement of the Lemma. The second is a restatement of Lemma 2.11(b) of [2]. The third follows by a closure argument.

Finally we prove the estimate essential for the core property.

Lemma 2.4. If $f \in \mathscr{D}_{\infty} \cap D(\delta)$ then

$$
\lim _{\alpha \rightarrow 0}\left\|\left(\delta-\delta_{\alpha}\right) \tau_{t}^{\alpha} f\right\|=0
$$

uniformly for $t$ in bounded intervals.

Proof. First it follows from Lemmas 2.2 and 2.3 that $\tau_{t}^{\alpha} f \in D(\delta)$ $\cap D\left(\delta_{\alpha}\right)$. Now since $f \in \mathscr{D}_{\infty}$ there is an $m$ such that $s \rightarrow f\left(S_{s} \omega\right)$ is constant if $\nu(\omega) \geq m$, and hence $s \rightarrow\left(\tau_{t}^{\alpha} f\right)\left(S_{s} \omega\right)$ is also constant. Thus $\left(\delta_{0} \tau_{t}^{\alpha} f\right)(\omega)=0$ if $\nu(\omega) \geq m$. Moreover $\delta \tau_{t}^{\alpha} f=0=\delta_{\alpha} \tau_{t}^{\alpha} f$ on $X_{0}$. Therefore

$$
\begin{aligned}
\left\|\left(\delta-\delta_{\alpha}\right) \tau_{t}^{\alpha} f\right\| & \leq \sup _{\omega \in X \backslash X_{0}} \int d s g(s)\left|\lambda(\omega)-\lambda\left(S_{\alpha s} \omega\right) \|\left(\delta_{0} \tau_{t}^{\alpha} f\right)(\omega)\right| \\
& \leq \alpha K \int d s g(s)|s|\left\|\delta_{0} \tau_{t}^{\alpha} f\right\|
\end{aligned}
$$


where $K=\sup \{K(\nu(\omega)) ; \nu(\omega) \leq m\}$. Next we estimate $\left\|\delta_{0} \tau_{t}^{\alpha} f\right\|$ by use of the last statement of Lemma 2.3.

It follows easily that

$$
\left\|\delta_{0} \tau_{t}^{\alpha} f\right\| \leq\left\|\delta_{0} f\right\| \sup _{\substack{\omega \in X \backslash X_{0} \\ \nu(\omega) \leq m}} \exp \left\{\int_{0}^{t} d s \mid\left(\tau_{s}^{\alpha} \delta_{0} \lambda_{\alpha}\right)(\omega) \|\right\}
$$

and it remains to bound the exponential. But

$$
\begin{aligned}
\left|\left(\tau_{s}^{\alpha} \delta_{0} \lambda_{\alpha}\right)(\omega)\right| & \leq \lim _{h \rightarrow 0} \int d r g(r)\left|\left(\tau_{s}^{\alpha} \lambda\right)\left(S_{\alpha r+h} \omega\right)-\left(\tau_{s}^{\alpha} \lambda\right)\left(S_{\alpha r} \omega\right)\right| /|h| \\
& \leq K(\nu(\omega))\left\{1+\lim _{h \rightarrow 0} \int d r g(r)\left|x_{\omega_{1}}^{\alpha}(s)-x_{\omega_{2}}^{\alpha}(s)\right| /|h|\right\}
\end{aligned}
$$

where for brevity we have set $\omega_{1}=S_{\alpha r+h} \omega$ and $\omega_{2}=S_{\alpha r} \omega_{\text {。 Now note }}$ that $\nu\left(\omega_{1}\right)=\nu\left(\omega_{2}\right)=\nu(\omega)$ so the frequencies of all points involved are uniformly bounded by $m$. Next by the arguments given earlier in the section there is a $K_{\omega}<\infty$ such that

$$
\left|\lambda_{\alpha, \omega_{i}}(t)\right| \leq K_{\omega}(1+|t|)
$$

for $1>\alpha>0, i=1,2$, and all $t \in \mathbb{R}$. Next fix $t_{0}>0$ and define

$$
M_{\alpha r, h}=\sup \left\{\left|\lambda_{\alpha, \omega_{1}}(t)-\lambda_{\alpha, \omega_{2}}(t)\right| ;|t| \leq M_{\omega} t_{0}\right\}
$$

where

$$
M_{\omega}=\left(e^{K_{\omega} t_{0}}-1\right) / t_{0}
$$

Then it follows from the estimates used to prove Observation 1 in Section 2 of [2] that

$$
\left|x_{\omega_{1}}^{\alpha}(s)-x_{\omega_{2}}^{\alpha}(s)\right| \leq M_{\alpha r, h}\left(e^{M_{\omega}|s|}-1\right) / M_{\omega}
$$

But by the Lipschitz condition on $\lambda$

$$
M_{\alpha r, h} \leq K(\nu(\omega))|h| \text {. }
$$

Combining these estimates finally gives

$$
\left|\left(\tau_{s}^{\alpha} \delta_{0} \lambda_{\alpha}\right)(\omega)\right| \leq K(\nu(\omega))\left(1+K(\nu(\omega)) \mid\left(e^{M_{\omega}|s|}-1\right) / M_{\omega}\right.
$$

Thus we have established that $\left\|\delta_{0} \tau_{t}^{\alpha} f\right\|$ is bounded uniformly for $0<\alpha<1$ and $|t| \leq t_{0}$.

Consequently

$$
\lim _{\alpha \rightarrow 0}\left\|\left(\delta-\delta_{\alpha}\right) \tau_{t}^{\alpha} f\right\|=0
$$

uniformly for $|t| \leq t_{0}$. 
Now we are prepared to prove the core property.

Let $H$ denote the generator extension of $\delta$ and take $f \in D(H)$. Since $\mathscr{D}_{\infty} \cap D(\delta)$ is dense in $\mathscr{A}$, by Lemma 2.7 of [2], there is a family $f_{\beta} \in \mathscr{D}_{\infty} \cap D(\delta), \beta>0$, such that $f_{\beta} \rightarrow f$ as $\beta \rightarrow 0$. Next choose $h \in C^{\infty}(-1,1)$ to be a positive function with total integral one and define $h_{\gamma}$ by $h_{\gamma}(t)=\gamma^{-1} h\left(\gamma^{-1} t\right)$, for $\gamma>0$. Then $\tau_{h_{\gamma}}^{\alpha} f_{\beta} \in \mathscr{D}_{1} \cap D(\delta)$ by Lemma 2.3

$$
\begin{gathered}
\tau_{r_{r}}^{\alpha} f_{\beta}=\int d t h(t) \tau_{r t}^{\alpha} f_{\beta} \\
\underset{\alpha \rightarrow 0}{\longrightarrow} \int d t h(t) \tau_{r t} f_{\beta} \\
\underset{\beta \rightarrow 0}{\longrightarrow} \int d t h(t) \tau_{\gamma t} f \underset{r \rightarrow 0}{\longrightarrow} f .
\end{gathered}
$$

But

$$
\lim _{\alpha \rightarrow 0}\left\|\left(\delta-\delta_{\alpha}\right) \tau_{h_{\gamma}}^{\alpha} f_{\beta}\right\|=0
$$

as a consequence of Lemma 2.4 and

$$
\begin{aligned}
& \delta_{\alpha} \tau_{h_{\gamma}}^{\alpha} f_{\beta}=-\gamma^{-1} \int d t h^{\prime}(t) \tau_{\gamma t}^{\alpha} f_{\beta} \\
& \underset{\alpha \rightarrow 0}{\longrightarrow}-\gamma^{-1} \int d t h^{\prime}(t) \tau_{\gamma t} f_{\beta} \\
& \underset{\beta \rightarrow 0}{\longrightarrow}-\gamma^{-1} \int d t h^{\prime}(t) \tau_{\gamma t} f \\
&=\int d t h(t) \tau_{\gamma t} H f_{\gamma \rightarrow 0}^{\longrightarrow} H f^{\longrightarrow} .
\end{aligned}
$$

This establishes that $H$ is the closure of $\delta$ restricted to $\mathscr{D}_{1} \cap D(\delta)$.

Finally if $f \in \mathscr{D}_{1} \cap D(\delta)$ then $\sigma_{h_{\gamma}} f \in \mathscr{D}_{\infty}$. But it follows by repetition of the argument used to prove Lemma 2.3 that $\sigma_{h_{r}} f \in D(\delta)$ and

$$
\begin{aligned}
\left\|\delta \sigma_{h_{\gamma}} f-\sigma_{h_{r}} \delta f\right\| & \leq \sup _{\omega \in X \backslash X_{0}} \int d t h(t)\left|\lambda\left(S_{\gamma t} \omega\right)-\lambda(\omega)\right|\left|\left(\delta_{0} f\right)\left(S_{\gamma t} \omega\right)\right| \\
& \leq \gamma\left\|\delta_{0} f\right\| K
\end{aligned}
$$

where $K=\sup \{K(\nu(\omega)) ; \nu(\omega) \leq n\}$ and $n$ is chosen such that $t \rightarrow J\left(S_{t} \omega\right)$ is constant if $\nu(\omega) \geq n$. Thus

$$
\delta \sigma_{h_{\gamma}} f \rightarrow \delta f
$$

and this proves that $\mathscr{D}_{\infty} \cap D(\delta)$ is a core of $H$. 


\section{§3. Concluding Remarks}

Theorem 1.1 is slightly unsatisfactory because the Lipschitz property is a global smoothness condition. In principle the conclusions of the theorem should follow from smoothness of $t \rightarrow \lambda\left(S_{t} \omega\right)$ only in neighbourhoods of the zeros of $\lambda$. Recently Batty [1] has shown that the low frequency boundedness together with the hypothesis that $t \rightarrow \lambda\left(S_{t} \omega\right)^{-1}$ is not locally integrable on either side of any zero or at $\pm \infty$ is sufficient to ensure that $\delta$ has a unique generator extension $H$. Nevertheless it is unclear whether $\mathscr{A}_{\infty} \cap D(\delta)$ is a core of $H$ under this weaker hypothesis. This is however the case [1] if $X=\mathbb{R}$.

\section{Acknowledgements}

I am grateful to Charles Batty for several useful conversations concerning the subject matter of this note and that of his forthcoming article.

\section{References}

[1] Batty, C. J. K., Derivations on the line and flows along orbits, Edinburgh preprint, 1985.

[2] Bratteli, O., Digernes, T., Goodman, F. and D.W. Robinson, Integration in Abelian $C^{*}$-dynamical systems, Publ. RIMS, Kyoto Univ。, 21 (1985), 1001-1030. 
